gewiß wieder zu erkennen. Anch die Partialinfloreszenzen von Nerium Oleander L. gehören hieher. Von Asclepiadaceen fällt mir Calotropis procera $\mathrm{R}$. Br. ein, wo indessen die Verhältnisse wenig übersichtlich scheinen und einer genaueren Darstellung bedürfen.

Bezüglich der Plumier'schen Abbildung konnnen wir also feststellen, daß von den anscheinend gegenständigen Blüten stets die eine den Abschluß eines Schraubelsympodiums darstellt, die andere ein weiter nicht verzweigtes $\beta$-Achselprodukt der nächst höheren Sproßgeneration. Eine Klärung der verwandtschaftlichen Beziehungen der Gattung Ancchites Griseb. kann aus diesen Feststellungen noch nicht erwartet werden, da wir über die Blütenstände der Familie noch viel zu wenig wissen, und daher den systematischen Wert schon gar nicht beurteilen können. Wobl aber ist festzustellen, daß sich Anechites lappulacea (Lam.) Miers. ableitet von dichasial verzweigten Formen, bei denen die. Verarmung in der Richtung zum reinen Schraubelsympodium sich in der Weise vollzog, daß sich - soweit beobachtet - die $B$-Blüten zwar entwickeln, daß sie aber stets sterile Vorblätter haben. Darin haben wir ganz unzweifelhaft einen abgeleiteten Charakter zu erblicken, in welchem Herkunft und Richtung des phylogenetischen Vorganges genau und eindeutig zu erkennen ist.

Vorstehende Studie wurde teils in der k. k. Hofbibliothek in Wien, teils in der Botanischen Abteilung des k. k. Naturhistorischen Hofmuseums ansgeführt. Den Vorständen der genannten Institute, dem Direktor und Vizedirektor der Hofbibliothek, Herrn Hofrat Dr. Josef D o n ab a um and Herrn Prof. Dr. Josef Bick, sowie Herrn Direktor Dr. Alexander Z $\mathrm{Zhl}$ bru ckner spreche ich auch an dieser Stelle meinen verbindlichsten Dank für das gewohnte Entgegenkommen aus.

\title{
Zur Pilzflora von Vorarlberg und Liechtenstein II.
}

Von Dr. Josef Murr (Feldkirch).

Nachstehendes Verzeichnis schließst sich an meine erste, unter derselben Überschrift in der Österr. bot. Zeitschrift, Jahrg. 1916, S. 88-94 erschienenen Liste an und enthält die zur Bestimmung gelangten Funde der Jahre 1916 und 1917 - einige Ergebnisse aus dem heurigen Jahre wurden gelegentlich der Korrektur eingeschaltet -, wobei aber Standorte bereits früher aus dem Gebiete bekannter oder in meinen ersten Beiträgen veröffentlichter Arten größtenteils unberücksichtigt bleiben.

Osterr. botan. Zeitschrift, 1918, Heft 10-12. 
Eigene Bestimmungen nẹhmen diesmal einen größeren Raum ein, wobei ich mich außer an Rabenhorst-Winter besonders an das praktische und klare Werk A. Ricken, „Die Blätterpilze Deutschlands usw." (Leipzig 1915) hielt. Außerdem fand ich bei Hochw. G. Bresadola in Trient und Freund Jos. Pöll in. Innsbruck, in einigen mikroskopischen Angelegenheiten auch bei dem während des Krieges hier in Feldkirch verweilenden hochverdienten Àskomyzeten-Forscher Prof. P. Ferd. Theißen stets freundliche und ausgiebige Unterstützung. Natürlich konnte gleichwohl wiederum sehr vieles infolge nicht genügenden oder nicht genügend gut erhaltenen Materials einer sicheren Bestimmung nicht zugeführt werden. Alle Funde ohne Bezeichnung gehören dem Verf. an; nur in Fällen, wo technische Gründe es verlangten, erscheint die Chiffre (M) beigesetat. Besonders viele wertvolle Funde brachte wiederum mein unermüdlicher Mitarbeiter Gemeinderat Stanislaus Kaiser (K) in Frastanz zu, gar manches Neue und Bemerkenswerte unterbreitete mir eine stattliche Reihe von Pilafreunden und -freundinnen, wie die Lehrerinnen Olga Schranz, Johanna Hefel und Bertha Hofbaur, die Lehrer Karl Zerlauth, Josef Bickel (Frastanz), Johann Bernhard (Koblach), Alfons Kranz (Ruggell in Liechtenstein), Fidel Wilhelmi (Röns), Fachlehrer Karl Sehmid (Hohenems), die Professoren Josef Rauch, Protas Heinrich, P. Karl München, Gymn.-Direktor KarlWinter, Chorregent Anton Schmutzer, die Pfarrer Theodor Hartmann (Ludesch), Julius Amann (Fraxern), Guntram Nagel (Silbertal), † Br. Josef Türk S. J., Finanzsekretär Otto R. v. Köpf (Bregenz), Steueroberverwalter Leop. Scheel, Postoberoffizial Pilade Rizzi aus Rovereto, die pens. Finanzwach-Oberaufseher Gebhard Stark (Mäder) und Alfons Gantner, Kaufmann Heinrich Gamohn, k. k. Förster Hermann Hosp, Waldaufseher Josef Amann (Frastanz), Frl. Rosa Kurzemann (St. Anton in Montafon), Frau Lucie Goop und Frau Josefine Hermann (Frastanz), Frau Wwe. Dr. Malin (Satteins), die Studenten Karl Grill und Livio Visinthainer.

Das Zeichen * bedeutet, daß eine Art für Vorarlberg neu, aber bereits aus Nordtirol angegeben ist, mit $*$ sind die aus Tirol nur vom südlichen Teile und mit *** die von Tirol überhaupt noch nicht veröffentlichten Arten gekennzeichnet.

Die Ausbeute der zwei letzten Jahre beläuft sich auf 240 für Vorárlberg neue Arten, wovon 50 nur von Südtirol, 70 von Tírol überhaupt nicht bekannt, bzw. veröffentlicht sind. Mit Einschluf der ersten Beiträge aus dem Jahre 1915 erreichen unsere bisherigen Neufunde für Vorarlberg die Zahl 380 (davon 70 bisher nur aus Südtirol, 100 aus Tirol überhaupt noch nicht "veröffentlichte Arten). 


\section{Myxomycetes.}

*Tubulina cylindrica (Bull.) Maria Grün, an faulen Strünken. Feldkirch.

* Trichia favoginea (Batsch) Pers. Verbreitet in Wäldern um

* Stemonitis sp. Eine wegen zu starker Eintrocknung und Überwucherung mir Sporotrichium agaricinum leider nicht mebr sicher bestimmbare Art (die Gattung neu f. Vbg.) auf Astmoosen in Maria Grün.

\section{Basidiomycetes.}

\section{Dacmyomycetaceae.}

* Calocera cornea (Batsch) Fr. An Strünken am Ardetzenberg und gegen das Älple.

\section{Clavariaceae.}

Clavaria fusiformis Sow. Bergwiesen ober Fraxern. C. ardenia Sow. Bergwiesen ober Fellengatter. ${ }^{*} *{ }^{*} C$. luticola Lasch. Lehmiger Wegrand im Ambergerwald. $\%$. rufescens Schaeff. Steinwald, Reichenfeld, Fellengatter; Rellseck in Montafon. ${ }^{*} C$. fastigiata L. Letzebühel.

Sparassis crispa Fr. Ardetzenberg (Hefel); für Tirol ron Hellwe ger bei Jungholz a. d. bayr. Grenze gefunden.

\section{Thelephoraceae.}

Cyphella muscicola Fr. Am Stamme einer Robinie in Feldkirch. **Thelephora cristata Pers. Auf abgefallenen Buchenblättern am Ardetzenberg. **Th. anthocephala Fr. Göfnerwald (Schranz, Zerla th, M., K.), Bodenwald, Ardetzenberg. Th. pallida Pers. Ardetzenberg.

*:* Pterula multifida Fr. Ambergerwald gegen Rankweil.

* Stereum avellanum Fr. Amerlügen, Kaltenbrunnen in Montafon. *St. ochroleucum Fr. Feldkirch.

\section{Hydnaceae.}

**Irpex lacteus Fr. An Ulmen in Reichenfeld, an Strünken bei Ludesch. *** canescens Fr. Göfnerwald (Hosp).

Hydnum corallioides Scop. Ellhorn in Liechtenstein. ${ }^{*} H$. cyathiforme Schaeff. Bildstein b. Bregenz. **II. nigrum Fr. Göfnerwald (Schmutzer). *H. ferrugineum Fr. Ambergerwald. H. aurantiacum (Batsch) Pers. Ellhorn. *H. levigatum Sw. Frastanz (K.), Göfnerwald (Zerlauth). H. subsquamosum Batsch. Amerlügen (Hefel). * $H$. torulosum Fr. Feldkirch (Heinrich). 


\section{Polyporaceae.}

*** Daedalea confragosa (Bolt.) Frastanz, an Erle (K.). D. cinnabarina Secr. Bodenwald bei Frastanz (K.). D. unicolor (Bull.). Vorderälple.

Trametes serialis Fr. *var. scalaris (Pers.) Fr. Frastanz (K.). T. cinnabarina (Jacq.) Fr. Bartholomäberg (Ra uch).

* Poria rufa (Schrad.) Frastanz (K.).

Polyporus versicolor (L.) *ar. nigricans (Lasch.). Amberg und Ardetzenberg, Brunnenfeld b. Bludenz. *P. zonatus (Nees) Fr. Frastanz (K.), an einem Maulbeerbaum in Feldkirch, Steinwald. P. igniarius (L.). Fr. Auf Hippophae in der Alpenanlage der Stella matutina. P. applanatus (Pers.) Wallr. Frastanz (K.), St. Kornelien, an Juglans. *P. betulinus (Bull.) Fr. Frastafeders (K.), bisher nur von Liechtenstein bekannt. *P. lacteus Fr. Bodenwald. P. sulphureus (Bull.) Fr. An einem Tannenstrunk in Jagdberg. *** P. acanthoides Fr. Steinwald. *P. umbellatus Pers. Wolfurt b. Bregenz (v. Köpf). P. melanopus (Pers.) Fr. Frastanz (Bickel). P. squamosus (Huds.) Fr. Frastanz, auf Aesculus (Goop), Bregenz ( $\nabla, K o ̈ p f)$. P. leucomelas (Pers.) Fr. Ludescherberg gegen den Frassen. $P$. ovinus (Schaeff.) Fr. Massenbaft in Silbertal in Montafon (Nagel). *P. subsquamosus (L.) Fr. (Valentin Nocker). - P. elegans (Bull.) Fr. var. nummularius (Bull.) Fr. Vaduz. P. arcularius (Batsch) Fr. Frastanzer Au. *** P. dichrous Fr. Ebenda an Erle.

Boletus cavipes (Opatowski) Kalchbr. Fraxern (Jul. A mann).

Boletus versipellis Fr. Kristberg (Rau ch), Klösterle a. Arlberg. B. porphyrosporus Fr. Kristberg (Rauch), Bregenz (v. Köpf). *B. viscidus L. Zuerst von mir am Ardetzenberg gef., Küblerwald b. Fellengatter (Jos. Amann), Steinwald, Ellhorn in Liechtenstein. *B. aereus Bull. Fellengatter-Amerlügen (Gamohn), Göfnerwald (M., Sebranz), Fraxern (Heinrich), Dünserberg. Ich bezeichne die in unserem Gebiete ausschließlich auftretende Unterart mit hell lederbraunem Hut und gelbem, bei Schnitt oder Bruch sich sofort lebhaft blau oder spangrün verfärbenden Fleische als $\boldsymbol{B}$. aerugineus mh. ${ }^{1}$ ). ${ }^{*} B$. spadiceus Schaeff. Ardetzenberg. B. variegatus Sw. Ebenda (H ofba u r, M.). B. granulatus L. Auf Bergwiesen, z. B. im Liechtensteinischen ober Trisen und Masescha neben $B$. elegans der häufigste, stellenweise fast einzige. Pilz. *B. flavus With. Trisenerwald (Hofbaur). Über die neue, schöne, der hiesigen Gegend, wie es scheint, eigentümliche Form B. edulis Bull. *** var. pseudopurpureus mh. mit braunpurpurnem Hute uud rosenrot überlaufenen Röhren (Göfnerwald, Ardetzenberg, zuerst von Olga Schranz gef.) berichte ich abgesondert. B. satanas

1) Differt a $B$. aereo typico pileo pallide bruneo, carne non alba flavescente sed flava cyanescente. 
Lenz ist, obwohl von Rick im Göfnerwald angegeben, für unser Gebiet noch keineswegs sicher nachgewiesen, zumal die Unterlage im Göfnerwald größtenteils aus Quellsandstein besteht. Dagegen ist hierzulande $B$. lupinus Fr., dessen Fleisch unter Umständen beim Bruch gleichfalls zuerst schmutzigrot anläuft, in mannigfaltigen Formen außerordentlich verbreitet.

\section{Agaricaceae.}

*Lenzites mollis Hfl. Frastanz (K.).

*Lentinus squamosus Schaeff. Säntis-Aussicht im Göfnerwald (Zerlauth), an morschen Schwellen der Hämmerle'schen Fabriksbahn. L. cochleatus (Pers.). Bodenwald (K.), Maria Grün und anderwärts bei Feldkirch (M., Hefel, Gamohn).

*Panus conchatus (Fr.) Fellengatter (Schranz).

*Marasmius ingratus (Schum.). Frastanz (R., Joh. A mann), Schlins (Rauch), Göfnerwald, Steinwald, Ellhorn in Liechtenstein. *M. cohaerens (Pers.). Göfnerwald (Zerlauth), Stadtschrofen, Steinwald, Gamperdona. M. scorodonius Fr. Steinwald, im Gebiete ziemlich selten. $M$. rotula (Scop.). Maria Grün, Ardetzenberg. $* * M$. ramealis (Bull.). Maria Grün. **M. epiphyllus Fr. Ardetzenberg. *M. oreades (Bolt.) Fr. Frastanz (K.), Göfnerwald.

***Nyctalis parasitica (Bull.). Steinwald, Göfnerwald. *N. asterophora Fr. Göfnerwald (Wilh elmi).

***Cantharellus glaucus Batsch. Auf einer Bergwiese in Fellengatter. * C. olidus Quél.; Göfnerwald. Die $1-3.5 \mathrm{~cm}$ hohen Exemplare zeigen durchwegs graurosenrote Fürbung. C. infundibuliformis (Scop.) *var. subramosus Bres. Göfnerwald. C. aurantiacus (Wulf.) Fr. Bodenwald, Küblerwald, Satteinserberg, St. Anton in Montafon.

*Russula adusta (Pers.). Göfnerwald, Steinwald, Ardetzenberg. *R. nigricans (Bull.). Steinwald (R., M.). *R. virescens (Schaeff.). Göfnerwald (M., Rizzi), Satteins (Malin), Steinwald. *R. furcata (Pers.). Ardetzenberg, Göfnerwald, Steinwald (M.), Schlins (Rau ch). $* R$. cyanoxantha (Schaeff.). Verbreitet, am Kamme des Hohen Gerach ober Alpila noch bei $1750 \mathrm{~m}$. ${ }^{*} R$. azurea (Bres.). Ardetzenberg, Ruine Tosters, Steinwald. *R. vesca (Fr.). Amberg, Göfnerwald, Ardetzenberg (M.), Maria Grün (Hefel). *R. integra (L.). Fr. Göfnerwald, Stadtschrofen, Furx. *R. Linnaei (Fr.) Fraxern, Furx, Satteins, Maria Grün. * $R$. aurata (With.). Verbreitet: Feldkirch, Bildstein, St. Anton in Montafon, Ellhorn in Liechtenstein. *** olivascens (Fr.). Ambergerwald. * $R$. badia Quél. Göfnerwald, Ardetzenberg, Satteinserberg* **R. puellaris (Fr.). Hofnerwald, Steinwald, Tosters. R. foetens (Pers.). Furx, Klösterle a. Arlberg, überhaupt auf Bergwiesen verbreitet. $* R$. 
fragilis (Pers.). Häufig; **var. violacea Quél: Göfnerwald. * R. emetica (Schaeff.). Häufig bis in dıe Alpen, z. B. Frassen, Fluhereckalpe bei Ebnit. *R. citrina Gillet. Bodenwald (K.), Göfnerwald (M., Zerlauth). R. rubra (Krombh.). Ardetzenberg, im Göfnerwalde auch die var. mit gedrängten Lamellen. - *R. delica (Vaill.) Fr. Noflerwald bei Feldkirch, Vaduz. *R. sardonia Fr. Göfnerwald.

*Lactarius glycyosmus (Fr.) Frastanz (K.). L. lignyotus (Fr.). St. Anton in Montafon. Im Steinwald bei Feldkirch fand ich eine im Habitus stark dem L. fuliginosus (Fr.) genäherte Zwischenart mit gleichfalls aderigem; doch im Verbältnis zur Höhe des Stieles viel breiterem, in der Mitte nicht gebuckeltem Hute, die ich in meiner Sammlung als ** L. Theissenii mb. bezeichnete ${ }^{1}$ ). L. hysginus Fr. St. Anton in Montafon, Bildstein. L. rufus (Scop.) Fr. Am Kristberg (Schmutzer, Winter) und in Monteneu bei Schruns noch bei $1400 \mathrm{~m}$. L. pallidus (Pers.). Fr. Ardetzenberg, Ludesch. L. acer (Bolt.) Fr. Göfnerwald, Bildstein. L. uvidus Fr. Bildstein. * L. blennius Fr. Ardetzenberg. *** L. circellatus (Batt.). Ebenda. *L. insulsus Fr. Hoch wald am Ludescherberg gegen den Frassen. ${ }^{2}$. jecorinus (Fr.). Fellengatter (M.), St. Anton in Montafon (Kurzemann). L. quietus (Fr.) ist vorläufig für unser Gebiet zu tilgen. Das in Formol bestens erhaltene Exemplar der Rick'schen Sammlung gehört zu L. volemus, der von Rick seltsamerweise nicht angegeben wird.

Hygrophorus pratensis Pers. Fellengatter, Furx bis $1170 \mathrm{~m} .{ }^{*} H$. niveus (Scop.). Fellengatter, zahlreich. H. caprinus (Scop.). Furx. ***H. ovinus (Bull.). Fellengatter. H. punicens (Fr.). Überall auf Bergwiesen, am Freschen bis $1800 \mathrm{~m}$. $*$ H. obrusseus (Fr.). Frastanz (K.). Fellengatter. $H$. chlorophanus (Fr.). Verbreitet auf Bergwiesen. *H. psittacinus (Schaeff.). Fellengatter. H. cerasceus (Wulf.). Ebenda.

Limacium erubescens (Fr.) ist für unser Gebiet noch nicht sicher. nachgewiesen und zumindest großenteils mit $L$. russula (Schaeff.) verwechselt; dagegen ist L. pudorinum (Fr.) ein (sehr gut eßbarer) Charakterpilz Vorarlbergs. L. capreolarium (Kalchbr.). Bildstein. *** L. leucophaeum (Scop.). Göfnerwald (Zerlauth) Maria Grün. * L. limacinum (Scop.). Moosige Bergwiesen ober Maria Ebene.

Gomphidius glutinosus (Schaeff.) ist im Gebiate häufiger als $G$. viseidus L. und geht z. B. am Dünserberg bis $1300 \mathrm{~m}$. *G. roseus (Fr.). Steinwald. *G. maculatus (Scop.) Fr. Maria Grün.

1) Differt a L. lignyoto p i le o multo la ti or e (L. lignyotus apud nos semper pileo pro caule procero parvo) n o $\mathrm{n}$ u $\mathrm{mb}$ o n at o. A $\mathcal{L}$. fuliginoso differt pileo venoso. limbo tenui denticulata lamellis albidis caule nigrescente; sporis a $L$. lignyoto non veidenter recedit. 
*Coprinus comatus (Fl. Dan.) Frastanz (K.) und auch. sonst ziemlich verbreitet. *C. niveus (Pers.). Göfnerwald, Tisis, Ardetzenberg. * C. lágópus (Fr.). Stadtpark in Feldkirch, Karina. *C. nyctemerus (Vaill.). Karina. *: C. extinctorius (Bull.). In der Höhlung eines Robinienstammes in Feldkirch. ${ }^{*}$ C. similis (BK.-Br.). Auf abgelagerter Erde im Garten der Kapuziner dahier. ${ }^{*}$ C. tergiversans (Fr.). Am Wege vor St. Kornelien. $\because C$. plicatilis (Curt.). Aus einem Blumenstocke mir von meiner Gattin Antonie überbracht. ${ }^{*}$ C. hemerobius (Fr.). Letzebühel, Alpenanlage der Stella matutina. * C. disseminatus (Pers.). Mehrfach in Feldkirch. *** C. subtilis (Fr.). Auf Mist in Karina und an der Alpenanlage der "Stella matutina".

**Paxillus griseotomentosus (Secr.). Mäder (Stark), Tostererwald. *P. leptopus Fr. Vor Kühbruck in Gamperdornatal an Felstitzen, Frastanzer Au. P. prunulus Scop. *var. orcella (Bull.) Fr. Stelfeder b. Nenzig.

Inocybe umbrina (Bres.) [an nova species? Bres. in litt.]. Frastanz (K.). *I. petiginosa (Fr.). Ardetzenberg. ** I. fulvella Bres. Ardetzenberg. * I. descissa (Fr.). Göfnerwald. * I. scabella (Fr.). Ardetzenberg, St. Kornelien.-I. geophylla (Sow.). Bildstein, Ellhorn in Liechtenstein. **I. hirtella (Bres.). Feldkirch. * I. obscura (Pers.). Laubwald an der Stella mutatina. I. cincinnata (Fr.). Feldkirch. *I. fastigiata (Schaeff.). Frastanz (K.), verbreitet um Feldkirch, Schnifnerberg, St. Anton in Montafon, Bildstein (M.), Bregenz (v. Köpf). I. Bongardii Weinm. Steinwald, Maria Grün, Ardetzenberg. I. caesariata (Fr.): St. Kornelien. Auferdem noch die bei Ricken nicht genannten Arten: $I$. eutheles (Berk. et $\mathrm{Br}$ ). Reichenfeld. ${ }^{*}$ I. muricellata Bres. Steinwald. ${ }^{*} I$. similis Bres. Zahlreich auf sandiger Anschwemmung in der Frastanzer Au. *** I. umbrinella Bres. Steinwald.

***Hebeloma radicosum (Bull.). Fellengatter, Ardetzenberg (M.); Frastanz (K.). *H. spoliatum (Fr.). Frastanz (K.), Göfnerwald. H. fusipes Bres. Göfnerwald (Schranz).

**Myxacium salor (Fr.). Ambergerwald. M. delibutum (Fr.). Kristberg $1400 \mathrm{~m}$ (Schmutzer). M. collinitum (Pers.). Schnifnerberg. **Phlegmacium caerulescens (Schaeff.). Reichenfeld, Ambergerwald, Satteins. *Ph. purpurascens (Fr.). Bodenwald (K.) und sonst um Feldkirch. *** Ph. cyanopus (Sekr.). Steinwald, Älple, Satteinserberg. **Ph. calochroum (Pers.). Ambergerwald. * Ph. variegatum (Bres.). Steinwald. *Ph. fulgens (Schw.). Frastanz (K.). *Ph. multiforme (Fr.). Steinwald, Fellengatter, St. Kornelien. Ph. orichalcum (Batsch). Hochwald am Frassen, Bildstein. Ph. variicolor (Pers.). Fellengatter, in prächtigen Hexenringen. ***Ph. largum (Buxb.). Frastanz (K.); verbreitet um Feldkirch, Klösterle am Arlberg. *Ph. varium (Schaeff.). Fellengatter 
(Scheel), Schlins (Rauch), Steinwald. *Ph. infractum (Pers.). Frastanz (K.). **Ph. vespertinum (Fr.). Ardetzenberg.

* Inotoma violaceum (L.). Bodenwald, Göfnerwald. I. alboviolaceum (Pers.). Göfnerwald, Steinwald. *I. malachium (Fr.). Frastanz (K.). *I. traganum (Fr.). Steinwald.

Dermocybc cinnabarina (Fr.). Mäder (Stark), der Standort "Göfnerwald“ gehört zu D. sanguinea (Wulf.); letztere auch im Hochwald am Frassen bei $1400 \mathrm{~m}$ und in Bildstein., D. cinnamomea (L.). In kleinen Exemplaren öfters an alten Strünken, besonders im Gebirge: Klösterle, Kristberg, Schutanna-Hinterberg. *D. cotonea (Fr.). Maria Grün. D. rhaphanoides (Pers.). Häufig: Ambergerwald, Steinwald, St. Kornelien.

*Telamonia hinnulea (Sow.). Frastanz (K,). **T. iliopodia (Bull.). Ambergerwald. *T. torva (Fr.). Göfnerwald (K.).

***Hydrocybe colus (Fr.). Bildstein. H. armeniaca (Schaeff.). Bregenz ( $\nabla$. Köpf), Bildstein. ***H. erugata (Weinm.). Göfnerwald (K.). *H. castanea (Bull.). Ambergerwald und sonst um Feldkirch. ***H. fasciata (Fr.). Frastanz (K.). $*$ H. saniosa (Fr.). Steinwald. H. leucopus (Bull.). Verbreitet. ***H, Krombholzii (Fr.). Steinwald. *H. obtusa (Fr.). Reichenfeld, Steinwald, Ardetzenberg.

* Pholiota praecox (Pers.). Verbreitet: Schallerhof am Ardetzenberg 8. I. 1916, auf Wiesen in Maria Grün durchgehends mit völlig verkümmertem Ring, Göfnerwald, Ambergerwald, gegen Übersaxen, im Buchenwald hinter Viktorsberg eine besonders große, bleiche Waldform. *Ph. adiposa (Fr.). Frastanz (K,). Ph. squarrosa (Fl. Dan.). Frastanz (Goop), Tschagguns (Schmid), Letze, Göfnerwald. *Ph. caperata (Pers.). Göfnerwald (Schranz), Ardetzenberg, St. Anton in Montafon, Bildstein (M.), Langenegg-Krumbach (v. Köpf), Bartholomäberg (Ra u ch), Kristberg (Schmutzer, Winter). *Ph. terrigena (Fr.). Göfnerwald. Ph. togularis (Bull.). Hochwald am Frassen bei $1600 \mathrm{~m}$.

* Flammula spumosa (Fr.). Göfnerwald. *** Flavida (Schaeff.). Steinwald. Fl. penetrans (Fr.). Steinwald. ***Fl. liquiritae (Pers.). Maria Grün.

**Naucoria lugubris Fr. Frastanz (K.), Steinwald (M., K.). *N. cucumis (Pers.). Steinwald, Göfnerwald. *N. pellucida (Bull.). Alpenanlage der Stella matutina (Türk).

*** Galera lateritia (Fr.). Verbreitet um Feldkirch auf Grasboden, Garten- und Schuttland; Lorüns b. Bludenz. *G. rubiginosa (Pers.). Steinwald, Karina. ***G. mniophila (Lasch.). Zahlreich auf sumpfigen Bergwiesen in Fellengatter. 
* Crepidotus variabilis (Pers.). Schloß Amberg. C. mollis (Schaeff.). Ardetzenberg (Rauch), Ellhorn in Lichtenstein, Dornbirn.

* Psalliota pratensis (Schaeff.). Rankweil (Rizzi). *P. silvatica (Schaeff.). Verbreitet um Feldkirch, wenn auch nirgends zahlreich.

*** Stropharia albonitens (Fr.). Unter Gebüsch am Finanzgebäude in Feldkirch ( $\nabla$ isinthainer).

*Hypholoma lacrimabundum (Bull.). Gartenland in Feldkireh, St. Kornelien. H. Candolleanum (Fr.). Verbreitet in und bei Feldkirch, oft neben Coprinus-Arten, Ebnit $1100 \mathrm{~m}$. ${ }^{*} H$. sublateritium ( $\mathrm{Fr}$.). Tosterer Wäldehen. *H. epixanthum (Fr.). Steinwald. *H. polytrichi (Fr.). Göfnerwald. H. hydrophilum (Bull.). Erastanzer Au.

*** Psilocybe merdaria (Fr.). Ardetzenberg, Kulmalpe b. Übersaxen, Alpila am Hohen Gerach, Nenzigasttal b. Klösterle $1600 \mathrm{~m}$. *** P. physaloides (Bull.). Frastafeders (K.), Fellengatter, Maria Grün, Letze, Steinwald. $*$ P. ericacea (Pers.). Waldmoor hinter dem Gasserplatz *P. foenisecii (Pers.). Karina, Ardetzenberg, Dünserberg $1200 \mathrm{~m}$.

** Psathyrella subatrata (Batsch). In einem Blumenkübel im Kapuzinerkloster in Feldkireh.

*** Panaeolus phalaenarum (Fr.). Nenzigastalpe gegen die Reutlinger Hütte $1900 \mathrm{~m}$. *P. campanulatus (L.). Kulmalpe bei Übersaxen (Kranz, M.), Hohe Kugel $1600 \mathrm{~m}$ (Zerlauth, M.), Hoher Frassen. Nenzigasttal am Arlberg $1500 \mathrm{~m}$ (M.), Gasserplatz b. Feldkirch (Prof. Dr. Dalla Fior VI. 1918).

**ntoloma madidum (ㅌr.). Felsenau-Fellengatter. **E. prunuloides (Fr.). Frastanz (K.), Feldkirch. **. rhodopolium (Fr.). Verbreitet b. Feldkirch: Ardetzenberg, Reichenfeld, Steinwald. E. speculum (Fr.). Ellhorn in Liechtenstein.

* Volvaria bombycina (Schaeff.) Fr. Nofels, an einem Brette.

**:Leptonia incana (Fr.). Letze, Frastanzer Au, Lorüns b. Bludenz, ob Bartholomäberg $1250 \mathrm{~m}$. * L. chalybaea (Pers.). Göfnerwald (M. Zerlauth). *L. lampropus (Fr.). Karina, Maria Ebene, Weideboden in Lorüns.

* Nolanaea vinacea (Scop.). Gampelin b. Frastanz. *N. mammosa (L.) Fr. Steinwald (Rizzi). ${ }^{*}$ Nolanaea staurospora Bres. Fellengatter.

**Eccilia Mougeotii (Fr.). Karina b. Feldkirch. ***E. rusticoides (Gill.). Letze. Alpenanlage der Stella matutina.

Amanitopis vaginata (Bull.) die var. fulva (Schaeff.) und plumbea (Schaeff.) verbreitet; ***ar. alba Fr. Göfnerwald, Karina.

Amanita phalloides (Fr.). Steinwald, Sattelberg b. Klaus. A. mappa (Batsch). Mäder (Stark). *A. strobiliformis (Vitt.). Zuerst von mir 
ober dem Schlosse in Vaduz, dann von Alf. Ga nttner im Ochsenried b. Bangs und im Schildriet b. Frastanz gef. A. muscaria (L.). Ein Exemplar mit völlig fleckenlosem, dabei orangetarbenem Hute vor Frastanz durch Frau Hermann übermittelt.

* Lepiota Friesii (Lasch.). Frastanz (K.), Altenstadt b. Feldkirch (M.), Bregenz (v. Köpf). L. procera (Scop.) Fr. Ludesch (Hartmánn) Stelfeder b. Nenzing (Hosp). L. clypeolaria (Bull.). Schlins (Rauch), Lorüns. L. amianthina (Scop.). Massenhaft auf den Bergwiesen in Fellengatter (M.), Kristberg (Winter). *L: granulosa (Batsch). Steinwald. L. carcharias (Pers.). Satteinserberg. L. haematites (Bk.-Bres.). Frastefeders (K.).

*Tricholoma aurantium (Schaeff.). Maria Grün (Hof ba ur), Spondawald und Göfnerwald (K.). * T. ustale (Fr.). Göfnerwald (K.. M.). * Th. coryphaeum (Fr.). Tosterer Wäldchen. *T. equestre (L.). Nur ein dürftiges Ex. im Göfnerwald (K.). $* T$. portentosum (Fr.). Ludesch (H a rtman n). T. tigrinum (Schaeff.). Frastanz (K:). *T. vaccinum (Pers.) Bregenz (v. Köpf). T. psammopus (Kalchbr.). Amerlügen (Hefèl). * T. ionides (Bull.). In einer f. gracilis im Walde gegen das Älple. ***T. inamoenum (Fr.). Göfnerwald, Steinwald. *T. Georgii (Clus.). Gurtis (Hefel), Letze. T. gambosum Fr. Wald bei der Haltestelle KlausKoblach gegen Götzis. ${ }^{* * T . ~ c n i s t a ~(F r .) . ~ F r a s t a n z ~(K .) . ~ * * T . ~ p a n a e o l u m ~}$ (Fr.). Ardetzenberg, Fellengatter. ${ }^{*} T$. glaucocanum Bres. Frastanz (K.). T. nudum (Bull.). Ardetzenberg. * T. arcuatum (Bull.). Stadtschrofen - Felsenau. * T. excissum Fr. Reichenfeld. ***T. sordidum (Fr.). Fellengatter. *T. connatum (Schum.). Hochwald gegen das Bödele. $*$ T. sudum (Fr.) Felsenau-Fellengatter.

*Clitocybe imperialis (Fr.). Göfnerwald (Schranz), Furx, Jagdberg (M.), Frastanz (K., Hosp), Ludesch (Hartmann), St. Anton in Montafon (Kurzemann). C. candida (Bres.). Blasenberg b. Feldkirch (Grill), Satteinserberg. C. catinus (Fr.). Mäder (Stark), Frastanzer Au (K.). *C. phyllophila (Pers.). Fellengatter (K.). C. cerussata (Fr.). Frastanz (K.). ${ }^{*}$ C. inversa (Scop.). Hofnerwald, Dünserberg. $*$. incilis (Fr.). Bregenz (v. Köpf). *C. geotropa (Bull.). Maria Grün (K., M.): C. popinalis (Fr.) Bres. Frastanz (K.). C. nebularis (Batsch). Gegen C. gangraenosa Fr. neigend, im Ambergerwald. *** concava (Scop.). Hofnerwald, Maria Grün. *** orbiformis (Fr.). Maria Ebene. C. suaveolens (Schum.). Verbreitet: Göfnerwald, Steinwald, Maria Grün. ${ }^{* *} C$. diatreta (Fr.). Frastanz (K., Jos. A mann). *C. ectypa (Fr.). In der var. infumata Bres. im Göfner- und Hofnerwald.

*Omphalia epichysium (Pers.). Alpenanlage der Stella mutatina. **: N. griseopallida (Desm.). Heideboden bei Hohenems. *O. pyxidata (Bull.) var. muralis Sow. Alpenanlage der Stella matutina. *O. hepatica 
(Batsch). Zwischen Moos in der Frastanzer Au (Dr. Dalla Fior u. M. 17. IV. 1918). *O. fibula (Bull.). Im Hochwald am Älple. ${ }^{* * *} 0$. bibula (Quél.). Steinwald. **o. camptophylla (Bk.). Alpenanlage der Stella matutina ( $\mathrm{Tür} \mathbf{k}$ ). ${ }^{*} O$. setipes (Fr.). Maria Grün, Steinwald. *O. plumbea (Fr.). Fellengatter.

**Collybia trigonospora.Bres. Ardetzenberg. **C. tesquorum Fr. (det. Bres., mir schienen die Exemplare gut zu C. ozes Fr. b. Ricken, Taf. 108. Fig. 9 zu stimmen). Göfnerwald, neben Baumwurzeln wie ausgesät. * $C$. longipes (Bull.). St. Kornelien (Hefel), Steinwald. C. butyracéa (Bull.). Göfnerwald, Steinwald, Bildstein. C. dryophila (Bull.). Ist im Gebiete doch vicht sehr häufig: Schnifis, Klaus; Ellhorn in Liechtenstein. ${ }^{*} C$. conigena (Pers.). Rankweil, Göfnerwald gegen Hofen. *C. esculentr (Wulf.). Ardetzenberg, Göfnerwad. C. cirrhata (Schum.). Steinwald; Fellengatter, Ardetzenberg. ${ }^{*}$ C. tuberosa (Bull.). Ardetzenberg.

*Mycena vulgaris (Pers.) Fr. Steinwald. *M. rorida (Fr.). Steinwald. *.1l. rosella (Fr.). Göfnerwald, Ambergerwald. *M. aurantiomarginata (Fr.). Maria Grün. **M. hiemalis (Osb.). An einer Robinie in Feldkirch 5. VIII. 1917, ebenda an einer Pyramidenpappel 1. XII. 1917. *** M. speirea (Fr.). Alpenanlage der Stella matutiua. * $M$. vitilis (Fr.). Göfnerwald, Fellengatter. $M$. pura (Pers.). Ellborn in Lichtenstein *M. flavo-alba (Er.). Ardetzenberg, Maria Griun. **M. lactea (Pers.). Ardetzenberg. * M. polygramma (Bull.). Steinwald. *M. galericulata (Seop.). Steinwald, Fellengatter, Hochwald am Frassen. *M. rugosa (Fr.). Auf einer alten Weide nächst der Haltstelle Tisis. - *M. luteo-alba (Bolt.) Er. Fellengatter. ${ }^{* *}$ MI. atrocyanea (Batsch) Fr. Ebenda.

*** Pleurotus dryinus (Pers.). Frastanz (K.). *P. corticatus Fr. var. tephrotrichus Fr. Göfnerwald (Bernhard, Schmutzer), Feldkirch.

\section{Hymenogastraceae.}

*Rhizopogon luteolus Fr. Im Walde ob der Letze. Rh. aestivus (Wulf.) Fr. Ardetzenberg:

\section{Iycoperdonaceae.}

Bovista nigrescens Pers. Karina-Tisis, Kulmalpe am Muttkopf und Dünserberg, Tschuggenalpe am Freschen, Gaflei bei Vaduz, Rarensburger Hütte am Schafberg ca. $2000 \mathrm{~m}$ (bei Rick von Vorarlberg mit?).

*Scleroderma vulgare Hornem. Ruine Tosters, Reichenfeld. S. bovista Fr. Göfnerwald, Steinwald. 


\section{Ascomycetes.}

\section{Bulgariaceae.}

Ombrophila strobilina (Alb. et Schw.) Rehm. St. Kornelien, Steinwald.

\section{Helotiaceae,}

** Sclerotinia Durieuana Tul. Zwischen Moos im Frastanzer Ried 17. V. 1917 (K. u. M.).

\section{Eupezizaceae.}

***Humaria leucoloma (Hedw.) Rehm. Auf bloßem Boden vor der Hl. Kreuz-Kapelle in Feldkirch. H. ollaris (Fr.) Cooke. Waldsehlucht über Emsreute gegen Ebnit.

* Aleuria pustulata (Hedw.) Bres. Bodenwald b. Frastanz.

Plicaria fimeti (Fuckel) Rehm. Fellengatter.

Pustularia coronaria (Jacq.) Rehm. var. macrocalyx (Riess) Cooke. Um Feldkireh fast Charakterpilz im Vorsommer; am Dünserberg noch bei $1100 \mathrm{~m}$.

Lachnea umbrata (Fr.) Phillipps. Waldsehlucht über Emsreute gegen Ebnit.

\section{Helvellaceae.}

*Helvella monachella (Scop.). Sandige Anschwemmungen der Ill vor Frastanz.

Gyromitra infula (Schaeff.) Quél. Wald ober Fellengatter und gegen die Felsenau.

\section{Fungi imperfecti.}

\section{Mucedinaceae.}

Sepedoniun chrysospermum (Bull.) Fr. Hänfig im Gebiete (die Standortsangaben sind bei Dalla Torre-Magnus, S. 537 ausgefallen).

*Botrytis epigaea Link. var. rosea. Mehrfach auf nacktem Waldboden bei Feldkireh.

****Verticillium agaricinum (Link) mit *** Mycogone rosea Link an einer jungen Amanita bei Feldkirch (Schmutzer). 